\title{
Two cases of myeloid sarcoma presented in rare locations mimicking lymphoma: case reports
}

\begin{abstract}
Background: Myeloid sarcoma (MS) is a rare malignant neoplasm composed of myeloid blasts occurring at an extramedullary site. In nearly half of the patients, it is misdiagnosed initially, the most common being lymphoma. Here, we reported two cases of MS with monocytic differentiation, morphologically mimicking lymphoma.

Case presentation

Case 1: A 62-year-old female with 6 year history of chronic myelogenous leukemia (CML), presented with one month history of central nervous system (CNS) symptoms. A magnetic resonance imaging (MRI) scan showed several bilateral frontal and left parietal dural based lesions. Intraoperative consultation for the biopsy from the left frontal dural based lesion favored hematopoietic neoplasm. A limited negative lymphoma panel excluded lymphoma by flow cytometry. MS was diagnosed on the excised left frontal dural based lesion. BCR-ABL1 fusion was detected by fluorescence in situ hybridization (FISH). Intrathecal chemotherapy, however, showed no improvement.
\end{abstract}

Case 2: A 29-year-old female with no significant past medical history presented with two month history of fatigue, fever, night sweats, weight loss (10lb in one month), and multiple enlarged lymph nodes. MS was diagnosed on the excised left anterior cervical lymph node that was initially thought to be a large cell lymphoma though. A concurrent bone marrow biopsy showed cytogenetically-normal AML with monocytic differentiation and mutated FLT3 tyrosine kinase domain. After systemic chemotherapy, no residual acute leukemia was detected. In addition, the sizes of the enlarged lymph nodes were significantly decreased.

\section{Conclusion}

We reported two cases of MS with monocytic differentiation, morphologically mimicking lymphoma. One case was a blastic transformation of CML presented in CNS. The other case was MS with concurrent AML presented in lymph nodes. Systemic chemotherapy is the recommended treatment for MS in addition to surgical resection, if surgical resection is necessary.

Keywords: myeloid sarcoma (MS), monocytic differentiation, systemic chemotherapy
Volume 3 Issue 3 - 2016

\section{Yan Liu MD, Laura Denham MD, Jun Wang \\ MD}

Department of Pathology and Human Anatomy, Loma Linda University Medical Center, USA

\section{Correspondence: Jun Wang, Department of Pathology, Loma Linda University Medical Center, I 1234 Anderson Street, Room 2516, Loma Linda, CA 92354, USA, Tel (909) 558-4000, Fax} (909) 558-0400, Email JWang@llu.edu

Received: October 05, 2016 | Published: December 19 , 2016
Abbreviations: MS, myeloid sarcoma; AML, acute myeloid leukemia; MPN, myeloproliferative neoplasm; MDS, myelodysplastic syndrome; CNS, central nervous system

\section{Introduction}

Myeloid sarcoma (MS) is a rare, extramedullary tumor mass consisting of myeloid blasts with or without maturation. MS could occur isolated, although most often precedes, relapse or coincides with acute myeloid leukemia (AML), or less commonly represents an acute blastic transformation of myelodysplastic syndrome (MDS), myeloproliferative neoplasm (MPN) or MDS/MPN. ${ }^{1-3}$ In cases with granulocytic differentiation, eosinophilic metamyelocytes, erythroid precursors, and megakaryocytes are helpful clues for diagnosis, whereas MSs with monocytic differentiation are more difficult to recognized and more easily to be misdiagnosed as lymphoma. The most common site of involvement by MS is the skin. ${ }^{2,4}$ Central nervous system (CNS) or lymph nodes involvement is relatively rare.
Here, we reported two cases of MS with monocytic differentiation. One involved CNS parenchyma; the other one involved lymph nodes.

\section{Case presentation}

\section{Case I}

A 62-year-old female with 6 year history of chronic myelogenous leukemia (CML), BCR-ABL1 positive, presented with one month history of difficulty speaking and right hand weakness. She was on Bosutinib. A magnetic resonance imaging (MRI) scan showed several bilateral frontal and left parietal dural based lesions, which were suspicious for metastatic tumors, lymphomas, and multiple meningiomas. Intraoperative consultation for the biopsy from the left frontal dural based lesion showed sheets of medium to large sized discohesive cells with high nuclear to cytoplasmic ratios, favoring hematopoietic neoplasm (Figure 1A). A limited flow cytometry lymphoma panel was performed, showing negativity for CD45, CD19, Kappa surface, and Lambda surface. The left frontal dural 
based lesion was excised, thereafter. Microscopically, the tumor was composed of dense sheets of tumor cells showing very high nuclear to cytoplasmic ratios, moderate pleomorphism, vesicular chromatin, and prominent nucleoli. Mitotic activity was high, and frequent apoptotic bodies were seen (Figure 1B). Immunohistochemistry (IHC) supported myeloid differentiation, with positivity for CD34, CD117, CD43, and CD68 (Figure 1C-F). However, CD45 was negative, and myeloperoxidase showed a weak blush of positivity. CD3, PAX 8 , and CD20 were negative. The overall findings supported a diagnosis of MS. Fluorescence in situ hybridization (FISH) studies showed $100 \%$ of nuclei had BCR-ABL1 fusion. Given the patient's history of CML, this represented a blastic transformation of her CML. In addition, approximately $70 \%$ of nuclei had three copies of RUNX1T1 (at 8q22), RARA (at 17q21.1), and RUNX1 (at 21q22). The presence of additional copies of probes on chromosomes 8, 17, and 21 suggested numeric or structural abnormalities of these chromosomes and indicated a more complicated leukemic clone. The patient was treated with intrathecal chemotherapy. A repeat MRI following the first course of intrathecal chemotherapy showed no residual tumor from the left frontal lesion, but persistence of the other lesions seen in the previous MRI and a new lesion in thoracic spine. Bone marrow biopsy, systemic chemotherapy and radiation were planned.
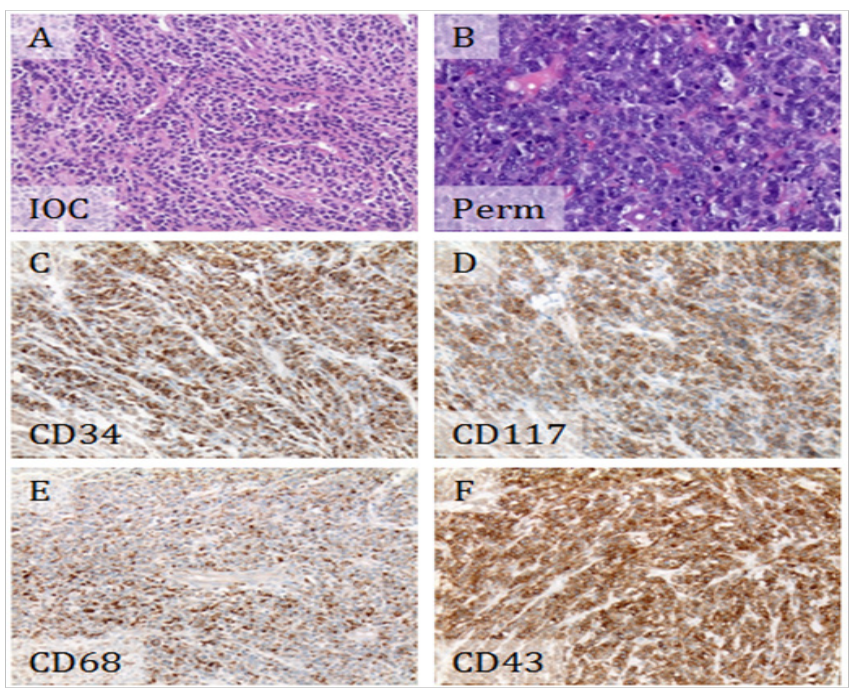

Figure I Myeloid sarcoma of case I (left frontal dural based lesion). An Intraoperative consultation tissue section (A) shows diffuse mononuclear blastic infiltrate (H\&E stain, 20x); Permanent section (B) show large blasts with prominent nucleoli (H\&E stain, 40x); The tumor cells are positive for blastic marker CD34 (C)(IHC stain, 40x); The blastic cells are also positive for myeloid marker CDII7 (D)(IHC stain, 40x) and weakly positive for monocytic marker CD68 (E)(IHC stain, 40x) and myelomonocytic marker CD43 (F)(IHC stain, 40x). H\&E, hemotoxylin \& eosin; IHC, immunohistochemistry.

\section{Case 2}

A 29-year-old female with no significant past medical history presented with two month history of fatigue, fever, night sweats, weight loss (10 lb in one month), and palpable neck lymph nodes. Physical exam showed multiple mobile non-tender enlarged lymph nodes in left anterior and posterior cervical and left axillary regions. Abdominal and pelvic CT scan showed multiple slightly enlarged lymph nodes in groin. Intraoperative consultation for the excised left anterior cervical lymph node showed that the normal nodal architecture was completely effaced by sheets of discohesive medium to large sized cells with high nuclear to cytoplasmic ratios and irregular nuclear contours (Figure 2A \& Figure 2B), suspicious for lymphoma. The cells on the permanent slides showed more morphologic details with reticular chromatin, prominent nucleoli, and moderate amount of foamy cytoplasm (Figure 2C \& Figure 2D). IHC supported myeloid differentiation, with positivity for CD117 and CD68 (Figure 2E \& Figure 2F), but negative for CD3 and PAX5. Immunophenotyping by flow cytometry showed an abnormal blast population $(\sim 69 \%$ total events) expressing CD13 dim, CD33, CD45 dim, CD56 partial, CD71 dim, CD117, and bright HLA-DR, but not expressing CD14 or CD34. The combined morphologic and immuno phenotypic features represented lymph node involvement by MS rather than a lymphoma. A concurrent bone marrow biopsy showed cytogenetically-normal AML with monocytic differentiation and mutated FLT3 tyrosine kinase domain. The MS is actually an extramedullary manifestation of her concurrent AML with monocytic differentiation. The patient was treated with systemic chemotherapy. Repeat bone marrow biopsies showed no residual acute leukemia. The sizes of the involved lymph nodes were significantly decreased.
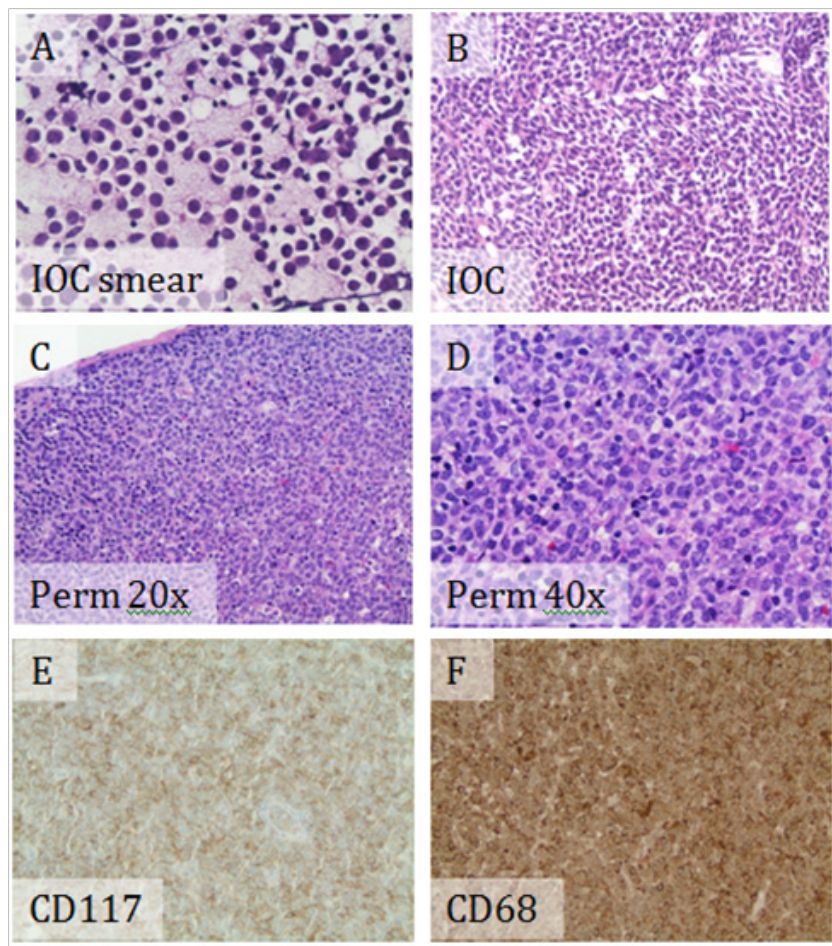

Figure 2 Myeloid sarcoma of case 2 (left anterior cervical lymph node). Both the Intraoperative consultation tissue smear (A)(H\&E stain, 40x) and the intraoperative consultation tissue section (B)(H\&E stain, 20x) shows numerous discohesive blasts; Permanent sections (C)(H\&E stain, 20x) and (D)(H\&E stain, 40x) show diffuse infiltrate of the similar large blasts; The blasts are positive for myeloid marker CDI 7 (E)(IHC stain, 40x) and myelomoocytic marker CD68

(F)(IHC stain, 40x). H\&E, hemotoxylin \& eosin; IHC, immunohistochemistry.

\section{Discussion}

MS is a rare extramedullary leukemic tumor comprising immature myeloid cells that disrupts the architecture of the tissues in which it is found. The tumor is also known as a chloroma due to its green color when exposed to air. This change occurs due to the presence of peroxidase, however, not all myeloid sarcomas are green and certain 
tumors are white in color. The etiology of MS is thought be due to aberrant homing signal for the leukemic blasts to localize in the bone marrow. ${ }^{2}$

MS can be divided into four subgroups: (1) isolated MS; (2) MS with concurrent AML; (3) extramedullary relapse of AML; and (4) blast phase/transformation of MDS, MPN or MDS/MPN. ${ }^{1}$ MS is more common in patients with AML with an incidence of $2.4-8 \% .^{5-7}$ In adults, roughly one third present with MS and concurrent myeloid disease (AML, MPN, and/or MDS) and one third have a history of treatment for a myeloid neoplasm. ${ }^{8}$ Thus, knowledge of the clinical history is extremely valuable in performing the appropriate workup and arriving at the correct diagnosis in these cases. Rarely, MS can occur in nonleukemic patients, and bone marrow studies did not reveal any hematological malignancies known as isolated MS. ${ }^{3}$ Most of these cases subsequently develop bone marrow involvement within a period of 1-year., ${ }^{910}$ However, in rare instances, cases have been reported in which transformation to leukemia has not occurred in a follow-up time of $>16$ years. ${ }^{11}$ The presence of MS is diagnostic of AML, regardless of the bone marrow or blood blast count. All attempts should be made to classify MS as performed in the bone marrow, with cytogenetic and immunophenotypic characterization.

MSs are most common in certain subtypes of AML, particularly M5a (monoblastic), M5b (monocytic), M4 (myelomonocytic), and M2 (myeloblastic with maturation). ${ }^{12}$ The diagnosis of MS may be challenging, especially if the patient had no history of leukemia. The tumors have the appearance of lymphomas and exhibit infiltrative growth without clear boundaries. In nearly half of the patients, it is misdiagnosed initially, the most common incorrect diagnosis being non-Hodgkin lymphoma (NHL). ${ }^{13}$ The accurate diagnosis is made by tissue biopsy with careful observation of tumor cells differentiating to granulocytes in mature stages and integration of these findings with the immunophenotypical staining on IHC, flow cytometry, and FISH. When biopsy samples are small and tissues are limited, touch preparations can be useful for cytochemical stains or FISH. The antigens most frequently expressed by MS include myeloperoxidase (MPO), CD68, lysozyme, CD43, CD117, CD11c, CD13, and CD33.,14 However, aberrant antigenic expressions were observed, such as CD4 and CD20. ${ }^{15,16}$ Differential diagnoses are broad and include NHL, Ewing sarcoma/primitive neuroectodermal tumors (PNET), carcinoma, etc., some of which cannot be correctly diagnosed until abnormal blood and bone marrow results are revealed.

Chromosomal aberrations are detected in about $55 \%$ of cases, including $\mathrm{t}(9 ; 22), \mathrm{t}(8 ; 21), \mathrm{t}(15 ; 17), \operatorname{inv}(16) / \mathrm{t}(16 ; 16), \operatorname{del}(5 \mathrm{q}),+8$, +21 , MLL-rearrangement, ect. ${ }^{4}$ For MS patients with AML associated with $\mathrm{t}(8 ; 21), \mathrm{t}(15 ; 17)$ or inv $(16) / \mathrm{t}(16 ; 16)$, a minimum blast count is not required, as is the case for bone marrow disease. However, this issue is less clear for AML with recurrent genetic abnormalities other than $\mathrm{t}(8 ; 21), \mathrm{t}(15 ; 17)$, or $\operatorname{inv}(16) / \mathrm{t}(16 ; 16) .{ }^{1}$ Regarding MS arising in patients with $\mathrm{CML}$, rare cases of BCR-ABL1-positive MS with no associated systemic disease have been described. ${ }^{17}$ The diagnosis of BCR-ABL1-positive AML/MS is currently controversial since others believe these cases are better classified as blast phase of CML. Detection of an NPM1 mutation, although not common, suggests de novo disease and supports BCR-ABL1-positive AML. ${ }^{18}$

Systemic chemotherapy, a regimen similar to that used to treatAML, is the current recommended treatment. ${ }^{2}$ Surgery and local radiation are considered to provide immediate symptomatic relief if the tumor causes organ dysfunction or obstructive symptoms. However, surgical resection and local radiotherapy cannot delay the transformation from myeloid sarcoma to AML or improve the prognosis. ${ }^{19}$ Even in isolated MS, AML-chemotherapeutic protocol is considered, even if surgical resections have been performed. It is because that the application of systemic chemotherapy soon after surgery is useful for controlling the development of the disease and improving the prognosis. ${ }^{20}$ Allogeneic hematopoietic stem cell transplantation (HSCT) combined with chemotherapy is also an effective treatment for MS. ${ }^{21,22}$

\section{Conclusion}

In conclusion, MS is a malignant neoplasm of myeloid origin that could develop in any area of the body. MSs derived from the CNS and lymph nodes are relatively rare and tend to be misdiagnosed, especially if they are with monocytic differentiation. High index of suspicion and appropriate myeloid markers by IHC and flow cytometry could come to the correct diagnosis. Intensive systemic chemotherapy with or without allogeneic HSCT should be performed in addition to surgical resection, local chemotherapy and radiotherapy.

\section{Acknowledgements}

None.

\section{Conflict of interest}

The author declares no conflict of interest.

\section{References}

1. Wilson CS, Medeiros LJ. Extramedullary manifestations of myeloid neoplasms. Am J Clin Pathol. 2015;144(2):219-239.

2. Avni B, Koren-Michowitz M. Myeloid sarcoma: current approach and therapeutic options. Ther Adv Hematol. 2011;2(5):309-316.

3. Yilmaz AF, Saydam G, Sahin F, et al. Granulocytic sarcoma: a systematic review. Am J Blood Res. 2013;3(4):265-270.

4. Pileri SA, Ascani S, Cox MC, et al. Myeloid sarcoma: clinico-pathologic, phenotypic and cytogenetic analysis of 92 adult patients. Leukemia. 2007;21(2):340-350.

5. Liu PI, Ishimaru T, McGregor DH, et al. Autopsy study of granulocytic sarcoma (chloroma) in patients with myelogenous leukemia, hiroshimanagasaki 1949-1969. Cancer. 1973;31(4):948-955.

6. Wiernik PH, Serpick AA. Granulocytic Sarcoma (Chioroma). Blood. 1970;35(3):361-369.

7. Neiman RS, Barcos M, Berard C, et al. Granulocytic sarcoma: A clinicopathologic study of 61 biopsied cases. Cancer. 1981;48(6):14261437.

8. Kjeldsberg CR. Practical diagnosis of hematologic disorders. USA: ASCP press; $2010.633 \mathrm{p}$.

9. Breccia M, Mandelli F, Petti MC, et al. Clinico-pathological characteristics of myeloid sarcoma at diagnosis and during follow-up: report of 12 cases from a single institution. Leuk Res. 2016;28(11):1165-1169.

10. Yamauchi K, Yasuda M. Comparison in treatments of nonleukemic granulocytic sarcoma. Cancer. 2002;94:1739-1746.

11. Movassaghian M, Brunner AM, Blonquist TM, et al. Presentation and outcomes among patients with isolated myeloid sarcoma: a Surveillance, Epidemiology, and End Results database analysis. Leuk Lymphoma. 2015;56(6):1698-1703. 
12. Byrd JC, Edenfield WJ, Shields DJ, et al. Extramedullary myeloid cell tumors in acute nonlymphocytic leukemia: a clinical review. J Clin Oncol. 1995;13(7):1800-1816.

13. Meis JM, Butler JJ, Osborne BM, Manning JT. Granulocytic sarcoma in nonleukemic patients. Cancer. 1986;58:2697-2709.

14. Amador-Ortiz C, Hurley MY, Ghahramani GK, et al. Use of classic and novel immunohistochemical markers in the diagnosis of cutaneous myeloid sarcoma. J Cutan Pathol. 2011;38(12):945-953.

15. Mourad W, Kfoury H, Al Husseini H. The value of CD34, myeloperoxidase and chloroacetate esterase (Leder) stain in the diagnosis of granulocytic sarcoma. Ann Saudi Med. 2001;21(5-6):287-291.

16. Bénet C, Gomez A, Aguilar C, et al. Histologic and immunohistologic characterization of skin localization of myeloid disorders a study of 173 Cases. Am J Clin Pathol. 2011;135(2):278-290.

17. Kuan JW, Pathmanathan R, Chang KM, et al. Aleukemic $<$ em $>$ bcr-abl $<$ em> positive granulocytic sarcoma. Leuk Res. 2016;33(11):1574-1577.
18. Konoplev S1, Yin CC, Kornblau SM, et al. Molecular characterization of de novo $\mathrm{Ph}+$ acute myeloid leukemia. Leuk Lymphoma. 2013;54(1):138144.

19. He J, Zhu L, Ye X, et al. Clinical characteristics and prognosis of nonleukemic myeloid sarcoma. Am J Med Sci. 2014;347(6):434-438.

20. Chelly I, Mekni A, Kchir N, et al. Intracerebellar granulocytic sarcoma: a case report. Pathologica. 2005;97(5):335-337.

21. Chevallier P, Mohty M, Lioure B, et al. Allogeneic hematopoietic stemcell transplantation for myeloid sarcoma: A retrospective study from the SFGM-TC. J Clin Oncol. 2008;26(30):4940-4943.

22. Yagi T, Ishikawa J, Takahashi M, et al. Successful treatment of duodenal myeloid sarcoma with allogeneic bone marrow transplantation and additional radiotherapy. Intern Med. 2012;51(7):769-772. 